\title{
2042 Reduction in pulmonary vein size after atrial fibrillation ablation in the absence of pulmonary vein stenosis Daniel Zalkind ${ }^{* 1}$, Subodh Joshi ${ }^{2}$, Allison Richardson ${ }^{2}$ and Anton Fuisz ${ }^{2}$
}

Address: ${ }^{1}$ Georgetown University Hospital, Washington, DC, USA and ${ }^{2}$ Washington Hospital Center, Washington, DC, USA

* Corresponding author

from I th Annual SCMR Scientific Sessions

Los Angeles, CA, USA. I-3 February 2008

Published: 22 October 2008

Journal of Cardiovascular Magnetic Resonance 2008, I0(Suppl I):A3 I I doi:I0.II86/I532-429X-I0-SI-A3 I I

This abstract is available from: http://jcmr-online.com/content/I0/SI/A3I I

(c) 2008 Zalkind et al; licensee BioMed Central Ltd.

\section{Introduction}

Pulmonary vein isolation (PVI) is an increasingly used treatment for atrial fibrillation. One serious complication of PVI is pulmonary vein stenosis (PVS). Even in patients without clinical PVS there may be a reduction in vein diameter post-procedure as measured on cardiac magnetic resonance imaging (MRI). It is unclear whether all four pulmonary veins decrease in size uniformly.

\section{Purpose}

To find statistically significant changes between the four main pulmonary veins after atrial fibrillation ablation.

\section{Methods}

Twelve consecutive patients with a mean age of 60 years old undergoing PVI by a single operator underwent cardiac MRI pre and post ablation. PVI was performed using standard ablation techniques and MRI scans were conducted on a 1.5 Telsa system with a protocol optimized for pulmonary venography using a 3D CE MRA sequence. All analysis was performed using a ViewForm workstation. All four pulmonary vein diameters were measured pre and post procedure.

\section{Results}

Three patients $(25 \%)$ had aberrant or accessory pulmonary veins. The median time between the pre-ablation and post-ablation scans was $151(+/-46)$ days. There was a significant reduction in pulmonary vein size post procedure of $1.5 \mathrm{~mm}(\mathrm{p}=0.002)$. Narrowing post-ablation was different between the four pulmonary veins $(p<0.05)$ with the left lower pulmonary vein showing greater change compared with the other three $(-3.3 \mathrm{~mm}$ vs $0.92 \mathrm{~mm}, \mathrm{p}=$ $0.013)$.

\section{Conclusion}

We found that even in patients without clinical pulmonary vein stenosis there is a reduction in vein diameter post ablation. There is a statistically significant difference in the narrowing between the four main pulmonary veins with the left lower pulmonary vein worst affected. 